\title{
Morphologic study of the effect of iron on pseudocyst formation in Trichomonas vaginalis and its interaction with human epithelial cells
}

\author{
Geovane Dias-Lopes ${ }^{1}$, Leonardo Saboia-Vahia ${ }^{2}$, Eliane Trindade Margotti ${ }^{3}$, \\ Nilma de Souza Fernandes ${ }^{3}$, Cássia Luana de Faria Castro ${ }^{4}$, \\ Francisco Odencio Oliveira-Junior', Juliana Figueiredo Peixoto', Constança Britto', \\ Fernando Costa e Silva-Filho ${ }^{6}$, Patricia Cuervo ${ }^{2}$, José Batista de Jesus ${ }^{1,3 /+}$
}

\begin{abstract}
'Fundação Oswaldo Cruz-Fiocruz, Instituto Oswaldo Cruz, Laboratório de Biologia Molecular e Doenças Endêmicas, Rio de Janeiro, RJ, Brasil ${ }^{2}$ Fundação Oswaldo Cruz-Fiocruz, Instituto Oswaldo Cruz, Laboratório de Pesquisa em Leishmanioses, Rio de Janeiro, RJ, Brasil ${ }^{3}$ Universidade Federal de São João Del Rei, Faculdade de Medicina, Departamento de Medicina, São João Del Rei, MG, Brasil ${ }^{4}$ Instituto de Ensino Superior Presidente Tancredo de Almeida Neves, São João Del Rei, MG, Brasil

${ }^{5}$ Fundação Oswaldo Cruz-Fiocruz, Instituto Oswaldo Cruz, Laboratório de Ultraestrutura Celular, Rio de Janeiro, RJ, Brasil

${ }^{6}$ Universidade Estadual do Norte Fluminense Darcy Ribeiro, Centro de Biociências e Biotecnologia, Laboratório de Biologia Celular e

Tecidual, Rio de Janeiro, RJ, Brasil
\end{abstract}

BACKGROUND Trichomonas vaginalis is the aetiological agent of human trichomoniasis, which is one of the most prevalent sexually transmitted diseases in humans. Iron is an important element for the survival of this parasite and the colonisation of the host urogenital tract.

OBJECTIVES In this study, we investigated the effects of iron on parasite proliferation in the dynamics of pseudocyst formation and morphologically characterised iron depletion-induced pseudocysts.

METHODS We performed structural and ultrastructural analyses using light microscopy, scanning electron microscopy and transmission electron microscopy.

FINDINGS It was observed that iron depletion (i) interrupts the proliferation of T. vaginalis, (ii) induces morphological changes in typical multiplicative trophozoites to spherical non-proliferative, non-motile pseudocysts, and (iii) induces the arrest of cell division at different stages of the cell cycle; (iv) iron is the fundamental element for the maintenance of typical trophozoite morphology; (v) pseudocysts induced by iron depletion are viable and reversible forms; and, finally, (vi) we demonstrated that pseudocysts induced by iron depletion are able to interact with human epithelial cells maintaining their spherical forms.

MAIN CONCLUSIONS Together, these data suggest that pseudocysts could be induced as a response to iron nutritional stress and could have a potential role in the transmission and infection of T. vaginalis.

Key words: Trichomonas vaginalis - iron - pseudocyst - morphological transformation - trophozoites - human epithelial cells

Trichomonas vaginalis is the aetiological agent of human trichomoniasis, which is one of the most prevalent sexually transmitted infections in humans. Trichomoniasis affects over 270 million people worldwide, and $90 \%$ of these infections occur among people living in resource-limited settings (WHO 2012). Although T. vaginalis infection is a commonly curable disease and treatment is available at an affordable cost, the emergence of drug resistance and the lack of alternative treatments are increasing concerns among public health policy makers (Meites et al. 2015).

Infection with $T$. vaginalis is associated with increased susceptibility to human immunodeficiency virus, herpes virus and papillomavirus infection, cervical cancer and prostate cancer. Particularly in women, infection of the urogenital tract may lead to pre-term delivery, low-weight births and infertility (Kissinger 2015, Meites et al. 2015).

doi: 10.1590/0074-02760170032

Financial support: FAPERJ (JCNE E-26/201·545/2014), CNPq

(Process no 308679/2012-1, 306393/2014-0, 305589/2015-16).

+ Corresponding author: jbj@ufsj.edu.br

Received 24 January 2017

Accepted 12 April 2017
During their life cycle, trichomonads present a trophozoite form without a true cystic stage. However, the presence of spherical forms with internalised flagella, denominated pseudocysts or endoflagellar forms have been commonly found in these parasites (Lipman et al. 1999, Granger et al. 2000, Pereira-Neves et al. 2003, 2011, 2012, da Costa \& Benchimol 2004, Ribeiro et al. 2015, Castro et al. 2016). Pseudocysts are observed mainly under unfavourable growth conditions and, at a low proportion, under in vitro axenic culture conditions (Pereira-Neves et al. 2003). In Tritrichomonas foetus, a trichomonad that infects the urogenital tract of cattle, pseudocysts induced by iron depletion are reversible and viable (Castro et al. 2016). In T. vaginalis, pseudocysts have been observed naturally in patients with cervical neoplasia (Afzan \& Suresh 2012) or induced in vitro by iron depletion (de Jesus et al. 2007).

In parasitic trichomonads, iron participates in the following: (i) sustaining the parasite's growth (Lehker \& Alderete 1992), (ii) modulating virulence (Kummer et al. 2008, Cárdenas-Guerra et al. 2013); (iii) hydrogenosomal metabolism via essential iron-sulfur [Fe-S] proteins (Vanáčová et al. 2001); (iv) regulating the expression of adhesins and cysteine peptidases involved 
in cytoadherence and cytotoxicity (Álvarez-Sánchez et al. 2007, Meza-Cervantez et al. 2011); (v) haemolysis (Cárdenas-Guerra et al. 2013); (vi) modulating the expression of ecto-ATPases and ecto-phosphatases (de Jesus et al. 2006); (vii) regulating Myb-like transcription factors (Hsu et al. 2012); (viii) inducing apoptosis in human epithelial cells (Kummer et al. 2008); and (ix) down-regulating the surface immunogen P270 (Alderete et al. 1999).

In previous studies, we observed that iron regulates protein expression in T. vaginalis and could be involved in the maintenance of the trophozoite morphology of this species (de Jesus et al. 2007). In the present study, we conducted a comprehensive morphologic analysis of the effect of iron on $T$. vaginalis. We used an approach that employs the removal of this metal from the culture medium by adding the iron chelator 2,2-dipyridyl. Thus, we investigated the effects of iron on parasite proliferation in the dynamics of pseudocyst formation and morphologically characterised the iron depletion-induced pseudocysts using structural and ultrastructural analyses by light microscopy, scanning electron microscopy and transmission electron microscopy. Furthermore, we evaluated the ability of the pseudocyst to interact with host cells and discussed the role of these forms in the life cycle of T. vaginalis and its relevance for the transmission and diagnosis of trichomoniasis.

\section{MATERIALS AND METHODS}

Chemicals - All the reagents were purchased from Sigma (St. Louis, MO, USA) or Merck (São Paulo, SP, Brazil). Milli-Q-purified water (Millipore Corp., Bedford, MA, USA) was used for all solutions. The iron chelator 2,2-dipyridyl is an organic, synthetic, membranepermeable compound that associates with extracellular and intracellular iron (Breuer et al. 1995).

Microorganisms and culture procedures - The FMV1 strain of $T$. vaginalis (de Jesus et al. 2004) was cultivated at $37^{\circ} \mathrm{C}$ in trypticase yeast extract maltose medium (TYM), $\mathrm{pH} 6.3$, supplemented with $10 \%$ heat-inactivated bovine serum and $0.6 \mathrm{mM} \mathrm{FeSO}_{4}$ (standard TYM medium). For pseudocyst induction, parasites cultivated in standard TYM medium were collected at the logarithmic phase of growth by centrifugation at $1400 \times \mathrm{g}$ for $5 \mathrm{~min}$ at $4^{\circ} \mathrm{C}$, washed twice with PBS, $\mathrm{pH} 7.2$, and incubated at $37^{\circ} \mathrm{C}$ for $48 \mathrm{~h}$ in TYM medium, $\mathrm{pH} 6.3$, supplemented with $10 \%$ heat-inactivated bovine serum and $180 \mu \mathrm{M}$ 2,2-dipyridyl (TYM-DIP inducer medium). The parasite densities and/or morphotypes were estimated by counts in a haemocytometer. In all assays, parasite viability was estimated using the Trypan blue dye exclusion test ( $0.4 \%$ in sterile PBS). Parasite cultures with viability above $90 \%$ were used.

Growth curve and effect of iron chelator on parasite proliferation - To evaluate the influence of iron chelation on parasite proliferation, $1 \times 10^{5} / \mathrm{mL}$ parasites were inoculated in $10 \mathrm{~mL}$ of standard TYM medium or TYMDIP inducer medium and were incubated at $37^{\circ} \mathrm{C}$ for 72 $\mathrm{h}$. The cellular density was evaluated daily by counting in a haemocytometer. Three independent assays were carried out in triplicate.
Parasite morphotypes and light microscopy analysis - The parasites were cultivated for $48 \mathrm{~h}$ at $37^{\circ} \mathrm{C}$ in standard TYM medium or TYM-DIP inducer medium. The counting of parasites morphotypes was performed every $12 \mathrm{~h}$ over $48 \mathrm{~h}$ in triplicate using a haemocytometer. For micrograph recording, the parasites cultivated in standard TYM medium or TYM-DIP inducer medium were harvested by centrifugation at $1400 \times g$ for $5 \mathrm{~min}$ at $4^{\circ} \mathrm{C}$, washed with PBS, pH 7.2, or PBS pH 7.2 containing $180 \mu \mathrm{M}$ 2,2-dipyridyl (PBS-DIP), respectively, and fixed in $2.5 \%$ glutaraldehyde. The parasite morphotypes were observed and counted by differential interference contrast microscopy (DIC) or on slides stained with the Panotico LB kit@(Laborclin, Brazil). Spherical, nonproliferative, non-motile parasites with all flagella internalised were considered as typical pseudocysts.

Analysis of the morphological reversibility of iron depletion-induced pseudocysts - To evaluate whether iron depletion-induced pseudocysts are viable and reversible forms, the parasites cultivated for $48 \mathrm{~h}$ in TYM-DIP inducer medium were washed twice with PBS-DIP, inoculated in fresh standard TYM medium and incubated at $37^{\circ} \mathrm{C}$ for $24 \mathrm{~h}$. Parasite morphology was examined by DIC, and the morphotypes were counted in a haemocytometer. Three independent assays were carried out in triplicate.

Scanning electron microscopy analysis - The parasites $\left(1 \times 10^{5} / \mathrm{mL}\right.$ cells) were inoculated in standard TYM medium or TYM-DIP inducer medium for $48 \mathrm{~h}$ at $37^{\circ} \mathrm{C}$. The parasites were then collected, washed and concentrated as described above, and a total of $5 \times 10^{6}$ parasites were fixed with $2.5 \%$ glutaraldehyde in $0.1 \mathrm{M} \mathrm{Na}$-cacodylate buffer $(\mathrm{pH} 7.2)$ at room temperature for $1 \mathrm{~h}$ at $25^{\circ} \mathrm{C}$ and post-fixed with a solution of $1 \% \mathrm{OsO}_{4}, 0.8 \%$ potassium ferricyanide and $2.5 \mathrm{mM} \mathrm{CaCl}_{2}$ in the same buffer for $1 \mathrm{~h}$ at $25^{\circ} \mathrm{C}$. The samples were dehydrated in an ascending acetone series and dried by the critical point method with $\mathrm{CO}_{2}$, mounted on aluminium stubs, coated with a 20-nm-thick gold layer and were examined with a Jeol JSM6390LV scanning electron microscope (Tokyo, Japan).

Transmission electron microscopy analysis - The parasites $\left(1 \times 10^{5} / \mathrm{mL}\right.$ cells) were inoculated in standard TYM medium or TYM-DIP inducer medium for $48 \mathrm{~h}$ at $37^{\circ} \mathrm{C}$. Thereafter, the parasites were collected, washed and concentrated as described above, and a total of $5 \times 10^{6}$ parasites were fixed with $2.5 \%$ glutaraldehyde in $0.1 \mathrm{M} \mathrm{Na}$-cacodylate buffer ( $\mathrm{pH} 7.2$ ) at room temperature for $40 \mathrm{~min}$ at $25^{\circ} \mathrm{C}$ and post-fixed with a solution of $1 \% \mathrm{OsO}_{4}, 0.8 \%$ potassium ferricyanide and $2.5 \mathrm{mM} \mathrm{CaCl}_{2}$ in the same buffer for 20 $\min$ at $25^{\circ} \mathrm{C}$. The parasites were then dehydrated in an ascending acetone series and were embedded in PolyBed 812 resin. Ultrathin sections were stained with uranyl acetate and lead citrate and were examined in a Jeol JEM1011 transmission electron microscope (Tokyo, Japan).

Effect of $\mathrm{FeSO}_{4}$ on iron depletion-induced pseudocysts - To demonstrate that the maintenance of the typical pear-shaped trophozoite morphology of $T$. vaginalis is dependent on iron, the parasites were cultivated for $48 \mathrm{~h}$ in TYM-DIP inducer medium, and then, $1.2 \mathrm{mM}$ $\mathrm{FeSO}_{4}$ was added and incubated for an additional $24 \mathrm{~h}$. 
Next, the morphotypes were determined by counting with a haemocytometer. Three independent assays were carried out in triplicate.

Analysis of T. vaginalis-HeLa cell interactions by scanning electron microscopy - HeLa cells (ATCC CCL2.1 HeLa 229) were cultured in 24-well plates in RPMI medium supplemented with $10 \%$ bovine serum and maintained at $37^{\circ} \mathrm{C}$ in an atmosphere of $5 \% \mathrm{CO}_{2}$ until confluence. Parasites cultivated in standard TYM medium or TYM-DIP inducer medium for $48 \mathrm{~h}$ at $37^{\circ} \mathrm{C}$ were submitted to interactions with a HeLa cell monolayer (5:1 parasites:HeLa cells) in RPMI medium supplemented with $10 \%$ heat-inactivated bovine serum and containing or not containing $180 \mu \mathrm{M}$ 2,2-dipyridyl. The interaction was allowed to proceed for $30 \mathrm{~min}$ at $37^{\circ} \mathrm{C}$ and $5 \% \mathrm{CO}_{2}$. To quantify adhered parasites, after $30 \mathrm{~min}$, the interaction assays were analysed using inverted phase-contrast microscopy with a $40 \mathrm{X}$ objective lens, and the percentage of adhered and non-adhered parasites was determined from counts of 400 parasites per well. Additionally, samples were fixed on slides and stained with the Panotico LB kit ${ }^{\circledR}$ to observe the morphology by light microscopy, or parasites were fixed and prepared for scanning electron microscopy analysis as described above.

Analysis of T. vaginalis-VEC interactions by light microscopy - Human vaginal epithelial cells (VECs) obtained from a young healthy donor were extensively washed with PBS pH 7.2 and resuspended in RPMI at $37^{\circ} \mathrm{C}$. Parasites $\left(1 \times 10^{5}\right.$ cells $)$ cultivated in $10 \mathrm{~mL}$ of standard TYM medium or TYM-DIP inducer medium were collected by centrifugation, washed in PBS or PBS-DIP, respectively, and then added to the VEC suspension in $500 \mu \mathrm{L}$ RPMI medium supplemented with $10 \%$ heat-inactivated bovine serum and containing or not containing $180 \mu \mathrm{M}$ 2,2-dipyridyl. The cell-parasite interaction was allowed to proceed for up $30 \mathrm{~min}$. To quantify adhered parasites, after the interaction, the cell suspension was homogenised and $50 \mu \mathrm{L}$ was distributed in microscopy slides (10 in total) and covered with coverslips, and the number of adhered parasites was estimated by counting in 20 random fields in each slide using DIC microscopy. In addition, samples were fixed on slides and stained with the Panotico LB kit $\AA$ to observe the morphology of adhered parasites using light microscopy.

\section{RESULTS}

Iron depletion inhibits T. vaginalis proliferation Comparison of the growth curves of $T$. vaginalis cultivated in standard TYM medium or TYM-DIP inducer medium for $72 \mathrm{~h}$ at $37^{\circ} \mathrm{C}$ showed that iron depletion inhibited parasite proliferation (Fig. 1). The parasites cultivated in standard TYM medium reached a log phase peak at $36 \mathrm{~h}$ with a maximum cell density of $1.7 \times 10^{6}$ cells $/ \mathrm{mL}$, followed by a strong decline in cell density (Fig. 1). In contrast, parasites cultivated in TYM-DIP inducer medium did not show significant growth but remained alive for up to $72 \mathrm{~h}$.

Light microscopy and ultrastructural analysis of the effect of iron depletion on the morphology of T. vagina-

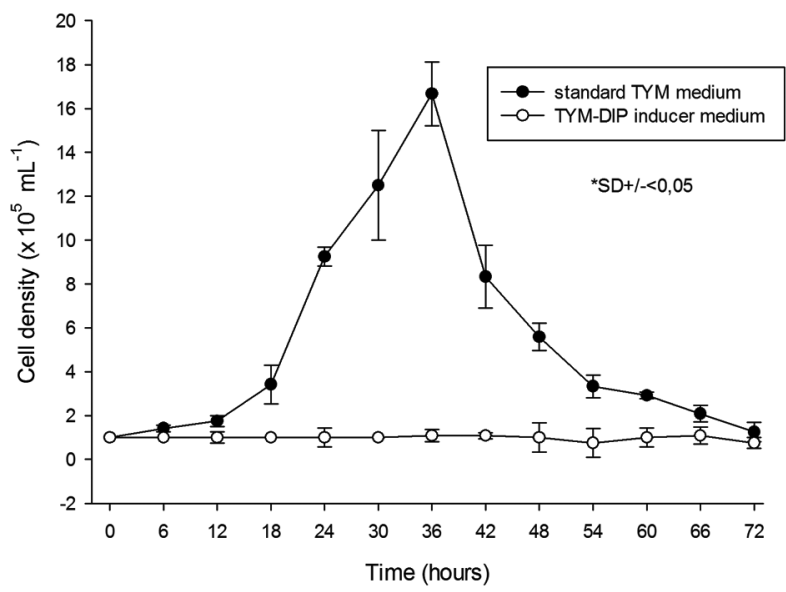

Fig. 1: effect of iron depletion on the cell growth of the Trichomonas vaginalis FMV-1 strain. Parasites were cultivated in standard TYM medium (filled circle) or TYM-DIP inducer medium (empty circle) for $72 \mathrm{~h}$ at $37^{\circ} \mathrm{C}$. The parasites were counted every $6 \mathrm{~h}$ using a haemocytometer. The experiments were performed in triplicate, and the results are shown as medians $\pm \mathrm{SD}$.

lis - Differential interference contrast microscopy (DIC) micrographs of parasites cultivated in standard TYM medium for $48 \mathrm{~h}$ showed typical pear-shaped trophozoite forms with four anterior flagella and a recurrent flagellum, an axostyle and an eccentric nucleus (Fig. 2A). Hydrogenosomes were clearly observed along the cell body (Fig. 2A, E). The parasites cultivated for $48 \mathrm{~h}$ in TYMDIP inducer medium exhibited typical spherical forms of different diameters, with flagella at the different stages of internalisation, slightly motile or non-motile (as observed previous to fixation), without a visible external axostyle (Fig. 2C). The process of flagellar internalisation of some cells seems to involve the internalisation of the recurrent flagellum first, followed by the anterior flagella. Typical spherical and non-motile pseudocysts with all internalised flagella were clearly observed (Fig. 2E).

Scanning electron microscopy of parasites cultivated in standard TYM medium for $48 \mathrm{~h}$ revealed typical trophozoites commonly found in axenic culture, exhibiting all anterior flagella and a recurrent flagellum associated with an undulant membrane (Fig. 2B). In contrast, parasites cultivated in TYM-DIP inducer medium for $48 \mathrm{~h}$ underwent a drastic morphological transformation from typical trophozoites to pseudocysts, which exhibit spherical forms, internalising anterior flagella and a recurrent flagellum (Fig. 2D, F). Some pseudocysts at different stages of flagella internalisation were also observed (Fig. 2D). Such observations were further corroborated by TEM analysis (Fig. 3). TEM clearly showed that parasites cultivated in TYM standard medium exhibited externalised flagella (Fig. 3A), whereas parasites cultivated in TYM-DIP inducer medium exhibited all internalised flagella (Fig. 3B).

Effect of iron depletion on the morphology of T. vaginalis - The examination of the morphology of the T. vaginalis population cultivated in TYM standard medium or 

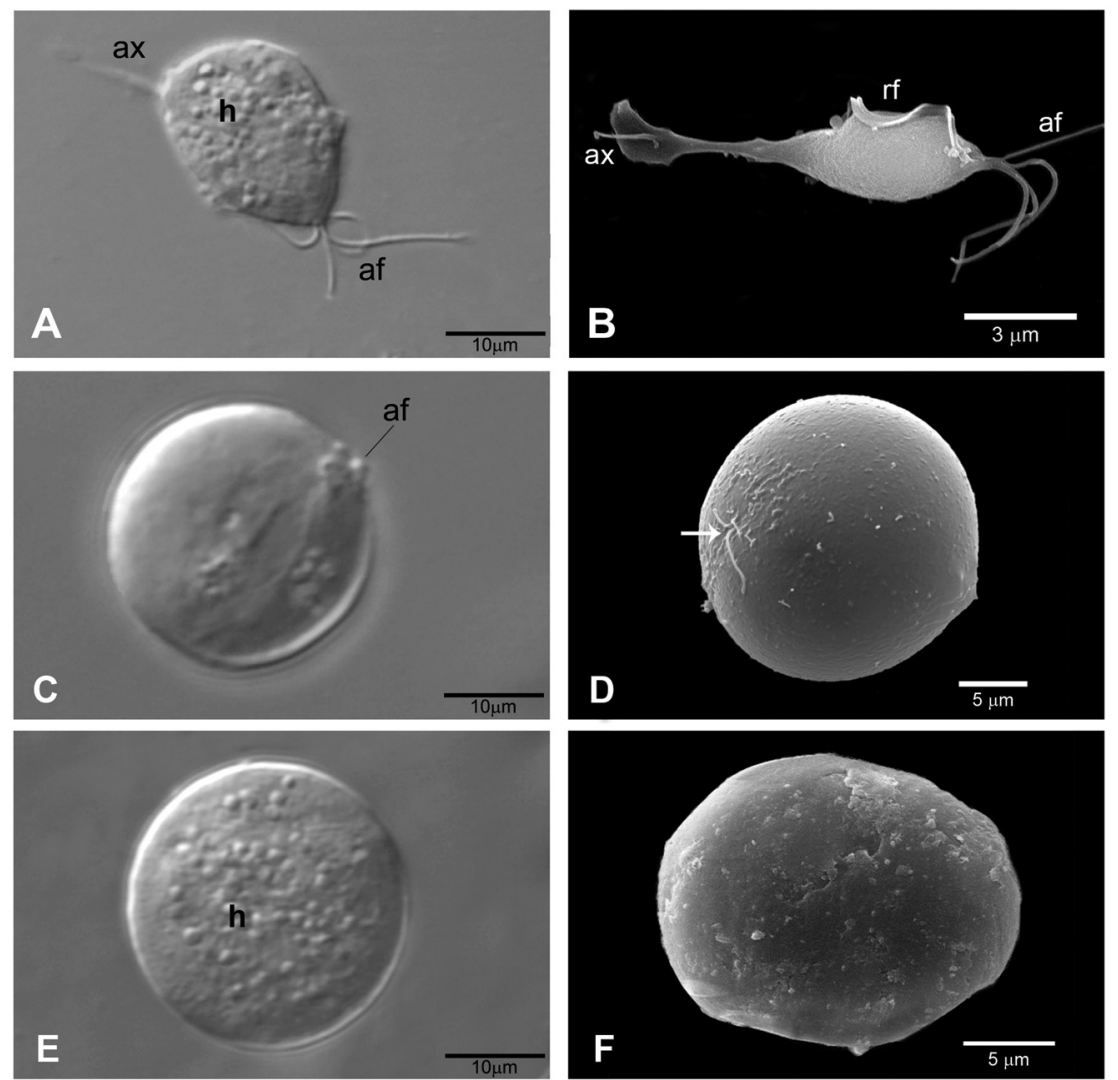

Fig. 2: effect of iron depletion on the morphology of Trichomonas vaginalis. Differential interference contrast microscopy (DIC) micrographs (A, C, E) and scanning electron microscopy (B, D, F) of T. vaginalis. Parasites cultivated in standard TYM medium for $48 \mathrm{~h}$ (A-B) show typical pear-shaped trophozoites with all flagella visible. Parasites cultivated in TYM-DIP inducer medium for $48 \mathrm{~h}$ (C-F) exhibited spherical forms at different stages of flagella internalisation $(\mathrm{C}, \mathrm{D})$, and typical pseudocysts with all flagella internalised (E, F). The arrow in D indicates the localisation of flagellar internalisation. ax: axostyle; af: anterior flagella; n: nucleus; h: hydrogenosomes.
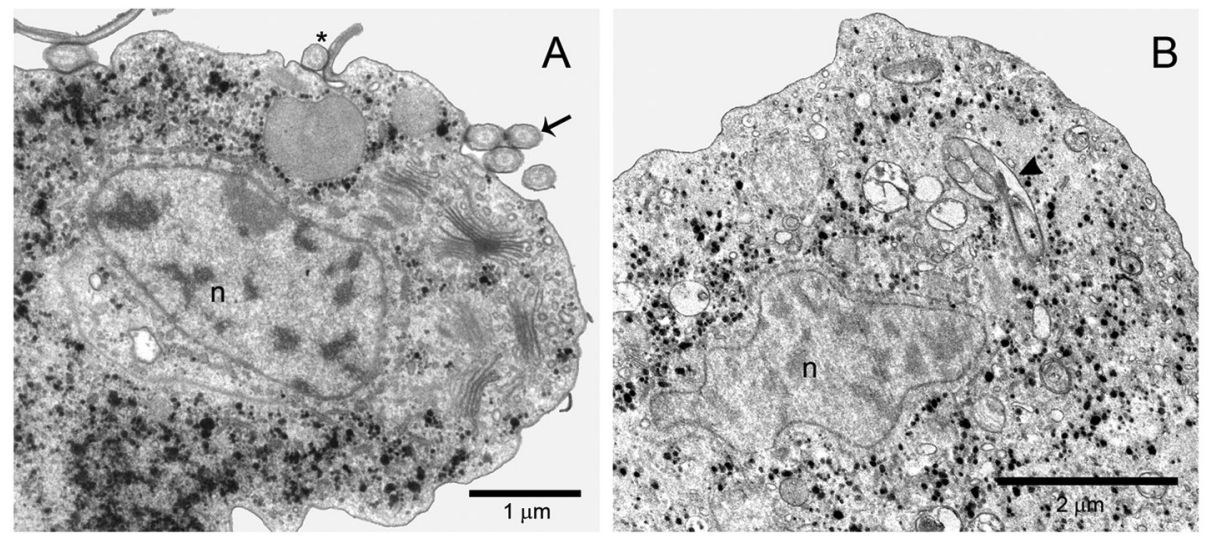

Fig. 3: transmission electron microscopy (TEM) of longitudinal thin sections of Trichomonas vaginalis. Parasites cultivated in either standard TYM medium for $48 \mathrm{~h}$ (A) or in TYM-DIP inducer medium for $48 \mathrm{~h}$ (B). (A) Typical trophozoite exhibiting externalised recurrent flagellum (*) and four anterior flagella structures (arrow). (B) Pseudocysts phenotype exhibit internalised flagella (arrow head). n: nucleus.

TYM-DIP inducer medium showed that iron depletion induced an increased percentage of pseudocysts over time compared with parasites cultivated in iron-rich medium (Fig. 4). In the control medium, approximately $95 \%$ of parasites presented the typical pear-shape motile trophozoite form during the experimental course (Fig. 4A). Remarkably, under this control condition, the parasite population contained nearly $5 \%$ pseudocysts (Fig. 4A). In contrast, in the TYM-DIP inducer medium, an increasing percentage of pseudocysts was observed over time (Fig. 4B). As early 

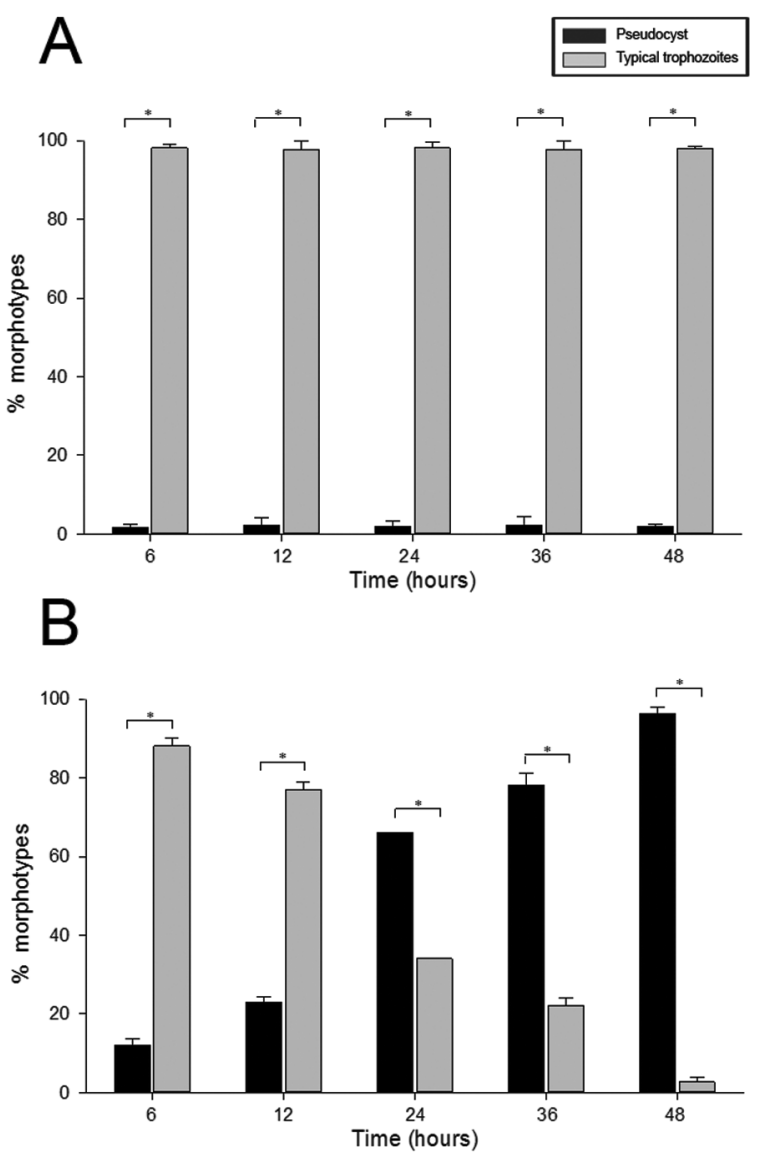

Fig. 4: effect of iron depletion on parasite phenotypes. Parasites were cultivated for $48 \mathrm{~h}$ in standard TYM medium (A) or TYM-DIP inducer medium (B). Counting of the parasite phenotypes was performed in triplicate using a haemocytometer. The results are presented as the means and standard errors from three independent experiments. *Significant differences between morphotypes $(\mathrm{p}<0.001)$.

as $6 \mathrm{~h}$ of culture, it was possible to observe $\sim 10 \%$ pseudocysts, and this number increased steadily over time, reaching $97 \%$ pseudocyst forms at $48 \mathrm{~h}$ (Fig. 4B).

Effect of iron depletion on the division process of $T$. vaginalis - The parasite population cultivated in standard TYM medium for $48 \mathrm{~h}$ exhibited cells in different phases of mitosis (Fig. 5A-D). In contrast, the cultivation of parasites in TYM-DIP inducer medium revealed parasites at different stages of flagella internalisation, showing a single nucleus or two nuclei and duplicated anterior flagella, indicating an interruption of the cell division process in different stages of the cell cycle (Fig. 5E, F).

Phenotype reversibility assay and the effect of $\mathrm{FeSO}_{4}$ on the morphology of iron depletion-induced pseudocysts - To analyse whether iron depletion-induced pseudocysts are viable cellular forms and whether such a phenotype is reversible to a typical trophozoite one, parasites cultivated for $48 \mathrm{~h}$ in TYM-DIP inducer medium were reinoculated into standard TYM medium and incubated for additional $24 \mathrm{~h}$. In this assay, a population of $T$. vaginalis cultivated for $48 \mathrm{~h}$ in TYM-DIP inducer medium and exhibiting ap- proximately 97\% pseudocysts (Fig. 6A, B) was inoculated into standard TYM medium; after $24 \mathrm{~h}$ of incubation, the morphotypes observed were mostly $(\sim 90 \%)$ typical trophozoites (Fig. 6A, C). The remaining $10 \%$ of the parasite population was composed of pseudocyst and parasite forms in the intermediate transformation process.

To evaluate whether iron supplementation could recover the trophozoite morphology and proliferation capability of iron-depletion-induced T. vaginalis pseudocysts, parasites cultivated for $48 \mathrm{~h}$ in TYM-DIP inducer medium were supplemented with $1.2 \mathrm{mM} \mathrm{FeSO}_{4}$ and were cultivated for an additional $24 \mathrm{~h}$. Morphotype evaluation showed that iron supplementation indeed recovered typical trophozoite morphology for $\sim 93 \%$ of the parasite population, whereas $\sim 7 \%$ maintained the pseudocyst form (Fig. 6D). This percentage of pseudocysts was similar to that of the pseudocyst population observed in the control (parasites cultivated in standard TYM medium).

Interaction of T. vaginalis with HeLa and vaginal epithelial cells (VECs) - Interaction of trophozoites or iron-depletion induced pseudocysts with HeLa cells was evaluated by SEM. Trophozoites exhibited high plasticity, yielding an amoeboid morphology after adhesion to the epithelial cells (Fig. 7A, B). Remarkably, pseudocysts were also able to adhere to HeLa cells, maintaining their spherical forms and emitting discreet membrane projections during contact with the host cell membrane (Fig. 7C, D, arrow).

Similar to that observed in the interaction with HeLa cells, trophozoites readily adhered to VECs, changing their typical ellipsoid forms to adherent amoeboid trophozoites (Fig. 8A, B). Amoeboid parasites exhibited multiple cytoplasmic projections, which mediated the parasite-VEC contact. In contrast, pseudocysts conserved their spherical forms during interaction with VECs but were able to adhere to the cells (Fig. 8C, D).

The percentages of adhesion of trophozoites and pseudocysts to HeLa cells were $74 \%$ and $16 \%$, respectively (Fig. 9A), whereas for VECs, the percentages of adhesion were $44.9 \%$ and $10,2 \%$ for trophozoites and pseudocysts, respectively (Fig. 9B).

\section{DISCUSSION}

In this study, we investigated the morphological alterations of $T$. vaginalis induced by iron depletion and verified the role of iron during the interaction of the parasite with epithelial cells. The results obtained here show that iron depletion (i) interrupts the proliferation of T. vaginalis; (ii) induces morphological changes in typical multiplicative trophozoites to spherical non-proliferative, non-motile pseudocysts; and (iii) induces the arrest of cell division at different stages of the cell cycle. Remarkably, we observed that (iv) iron is the fundamental element for the maintenance of typical trophozoite morphology; (v) pseudocysts induced by iron depletion are viable and reversible forms; and finally, we demonstrated that (vi) pseudocysts induced by iron depletion are able to interact with human epithelial cells, maintaining their spherical forms.

Here, we further analysed the effect of iron depletion on the morphological changes of trophozoites using structural and ultrastructural approaches. We observed 

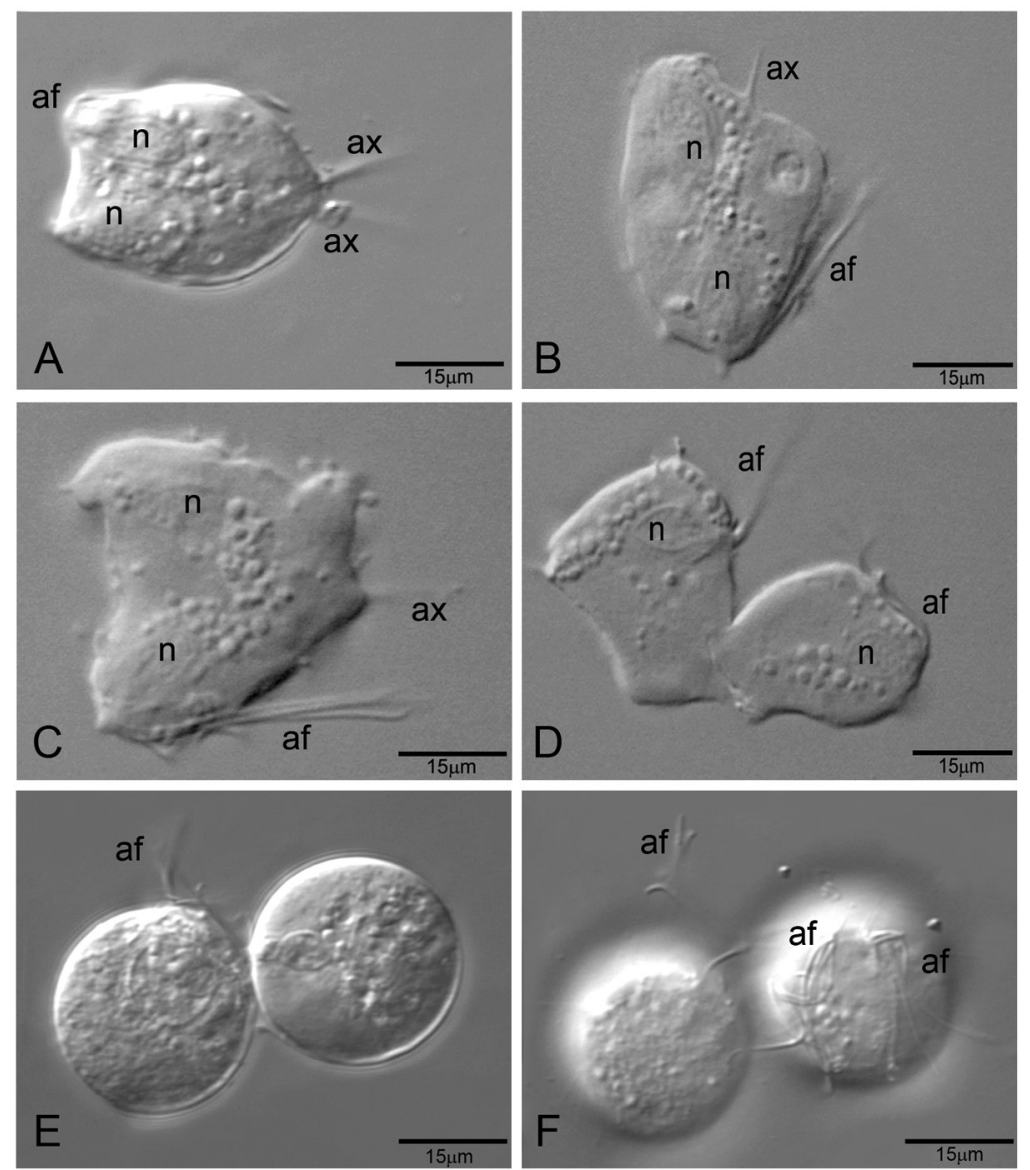

Fig. 5: effect of iron depletion on the division process of Trichomonas vaginalis. Differential interference contrast microscopy (DIC) of T. vaginalis cultivated in standard TYM medium for $48 \mathrm{~h}$ (A-D) showed that trophozoites underwent the cell division process; parasites cultivated in TYM-DIP inducer medium for $48 \mathrm{~h}(\mathrm{E}, \mathrm{F})$ showed parasites with arrested cell division. ax: axostyle; af: anterior flagella; n: nucleus.

that the inoculation of the trophozoite population from an iron-rich standard medium into an iron-depleted medium immediately interrupts parasite proliferation and induces concomitant morphological transformation to pseudocysts, which are parasite forms without apparent motility. Such a result shows that pseudocysts are non-replicative forms. Accordingly, the previous results of our group showed that iron depletion from $T$. vaginalis culture medium leads to a significant reduction in the expression of crucial proteins involved in the regulation of the cell cycle, protein synthesis, proteolysis and hydrogenosomal metabolism (de Jesus et al. 2007). Drastic alterations in protein synthesis, low metabolic activity and pseudocysts being non-motile parasites support the idea that pseudocysts are the non-replicative form of $T$. vaginalis under iron-depletion conditions. Although several studies have shown the deleterious effect of iron depletion on T. vaginalis proliferation and other parasites (Lehker \& Alderete 1992, Melo-Braga et al. 2003, de Jesus et al. 2006, 2007, Mesquita-Rodrigues et al. 2013, HernándezCuevas et al. 2014, Olmo et al. 2014; Thipubon et al. 2015, Castro et al. 2016), the association of iron depletion with morphological alteration from trophozoites to pseudocysts has not been assessed. Thus, we demonstrated that, in addition to its role in the regulation of parasite pro- liferation, iron also regulates parasite morphology. Because 2,2-dipyridyl is a lipophilic chelator that chelates iron from both the extracellular and intracellular milieu, the interruption of parasite proliferation could be due to (i) the interruption of the transcription of genes whose promoters are dependent on iron and/or (ii) the decreased or disrupted synthesis of essential [Fe-S] proteins that are crucial for the metabolism of parasites (Vanáčová et al. 2001, Hsu et al. 2012, Beltrán et al. 2013).

We observed that pseudocysts exhibit up to two nuclei. We believe that such an observation is not due to pseudocyst division but to the interruption of the cell division of parasites when they were inoculated into the TYMDIP inducer medium - i.e., when log-phase trophozoites undergoing division in the pre-inoculum standard TYM medium were inoculated in TYM-DIP inducer medium, they suffered arrest of the cell cycle and concomitantly suffered transformation to pseudocysts. We corroborated that pseudocysts are non-proliferative forms by the lack of an increased cellular density during the growth curve assay in TYM-DIP inducer medium. Bi- and multinucleated pseudocysts have also been observed in T. vaginalis (Abonyi 1995) and T. foetus (Pereira-Neves \& Benchimol 2009), including in fresh preputial smegma from infected bulls (Pereira-Neves et al. 2011). 

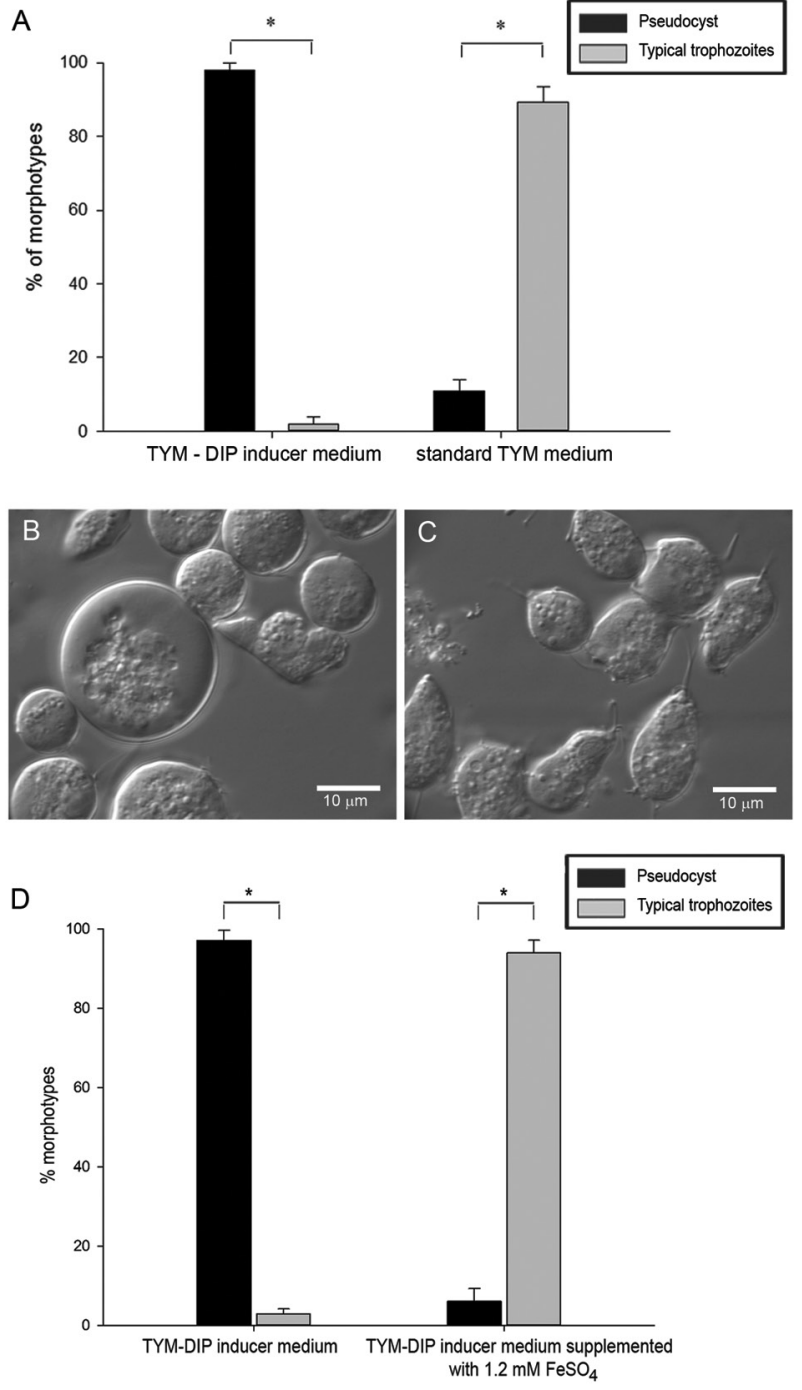

Fig. 6: phenotype reversibility of iron depletion-induced pseudocysts. (A) Parasites maintained for $48 \mathrm{~h}$ in TYM-DIP inducer medium (left) were collected, washed in PBS, and subsequently inoculated in fresh standard TYM medium (right) and incubated for $24 \mathrm{~h}$. (B) DIC of Trichomonas vaginalis cultivated in TYM-DIP inducer medium for 48 h. (C) DIC of $T$. vaginalis maintained for $48 \mathrm{~h}$ in TYM-DIP inducer medium and subsequently inoculated in fresh standard TYM medium and incubated for $24 \mathrm{~h}$. The micrographs show pseudocysts of different sizes (B) or typical pear-shaped trophozoites (C). (D) Effect of $\mathrm{FeSO}_{4}$ on the morphology of iron depletion-induced pseudocysts. Morphotypes of $T$. vaginalis cultivated in TYM-DIP inducer medium for $48 \mathrm{~h}$ (left), or TYM-DIP inducer medium for $48 \mathrm{~h}$ and then were supplemented with $1.2 \mathrm{mM} \mathrm{FeSO}_{4}$ and cultivated for an additional $24 \mathrm{~h}$ (right) were estimated by counting in a haemocytometer. The data obtained from three independent experiments are presented as the means \pm standard error. *Significant differences between morphotypes $(\mathrm{p}<0.001)$.

Trophozoites undergo a transformation from ellipsoid or amoeboid forms to rounded cells in which the flagella are internalised and axostyles are retracted. The formation of pseudocysts occurs in other trichomonads, including T. tenax (Ribeiro et al. 2015) and T. foetus, when subjected to distinct physical or chemical stimuli in the medium (Granger et al. 2000, Pereira-Neves et al.
2003, da Costa \& Benchimol 2004). We previously observed that the alteration of parasite morphology due to iron depletion was followed by a significantly different expression of cytoskeleton-related proteins and proteins involved in cell signalling (de Jesus et al. 2007). The cell surface of trichomonads exhibits an active endocytic machinery (Benchimol et al. 1986, 1990) and it has been suggested that during pseudocyst formation, the flagella are internalised by a receptor-mediated endocytosis process (Granger et al. 2000, Pereira-Neves et al. 2003). In addition, we observed that the sequence of flagellar internalisation was similar to that observed in T. foetus, where the recurrent flagellum is internalised first, followed by the anterior flagella (Pereira-Neves et al. 2003). Accordingly, the expression of several proteins involved in membrane remodelling and phagocytosis were modulated by iron depletion in T. vaginalis (de Jesus et al. 2007).

We observed that pseudocysts from TYM-DIP inducer medium were able to recover their trophozoite form and resume proliferation after re-inoculation in fresh standard TYM medium. Such results demonstrated that pseudocysts induced by iron depletion are viable and reversible forms. Accordingly, other authors have shown that pseudocysts of trichomonads represent a reversible form of the parasite that may occur as a response to drastic changes in the environment (Lipman et al. 1999, Pereira-Neves \& Benchimol 2009). We also observed that iron is the key regulator of trophozoite morphology because the addition of $\mathrm{FeSO}_{4}$ to TYM-DIP inducer medium - i.e., chelator-containing medium - reverted pseudocysts to trophozoites and recovered the proliferation capability of the parasite. Additionally, it has been shown that $T$. vaginalis pseudocysts occur naturally in the host, suggesting that these forms may coexist with replicative trophozoites (de Jesus et al. 2004, Afzan \& Suresh 2012). The natural occurrence of T. foetus pseudocysts was also observed in fresh bull preputial secretion, exhibiting a higher proportion of pseudocysts than that observed in culture in vitro (Pereira-Neves et al. 2011).

We also evaluated the capability of the pseudocysts to interact with human epithelial cells. In fact, we observed that although the adhesion percentage of pseudocysts was lower than that for trophozoites, pseudocysts induced by iron depletion were able to interact and adhere to HeLa cells and VECs. Such results, together with the previous observations that pseudocysts are found naturally in the host (de Jesus et al. 2004, Afzan \& Suresh 2012), suggest that pseudocysts could persist in the vaginal environment by adhering to host epithelial cells to avoid being carried out by menstrual fluid, for example. Alternatively, during the parasite life cycle, pseudocysts could be released during vaginal discharge, which could favour their dispersion and successful infection. Therefore, knowledge about the biology of pseudocyst formation is important not only to better understand the life cycle of $T$. vaginalis but also to understand their role in the dynamics of parasite transmission. Currently, in many countries, including Brazil, the diagnosis of trichomoniasis is based only on the detection of motile trophozoites in fresh clinical specimens; therefore, false-negative diagnoses may occur during the 

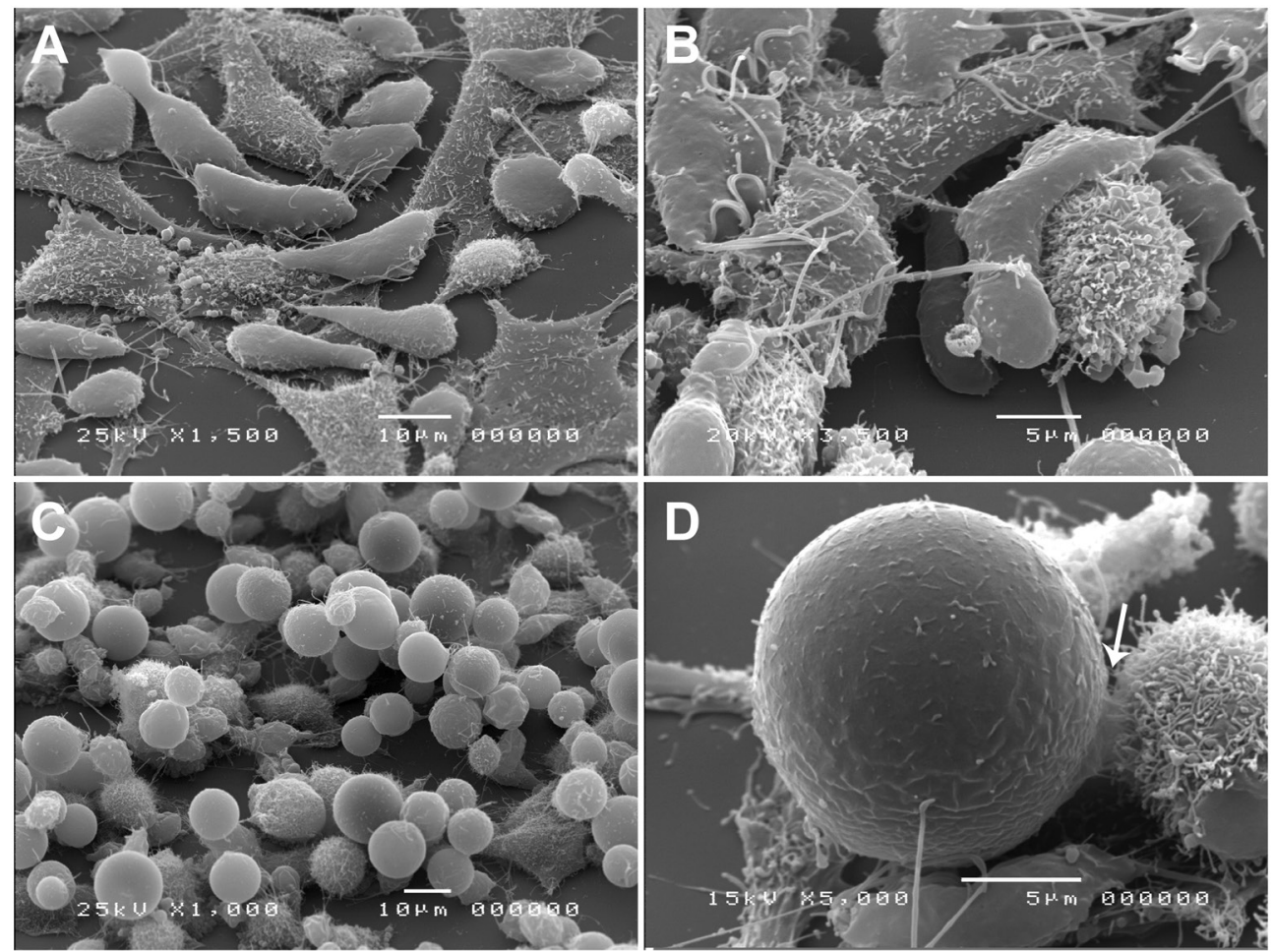

Fig. 7: Trichomonas vaginalis-HeLa cell interactions. Representative scanning electron microscopy (SEM) images of the morphology of trophozoites (A-B) or iron-depletion induced pseudocysts (C-D) during interaction with the HeLa cell monolayer. (C) A cluster of pseudocysts was observed. The arrow indicates membrane projections during contact with the host cell membrane.
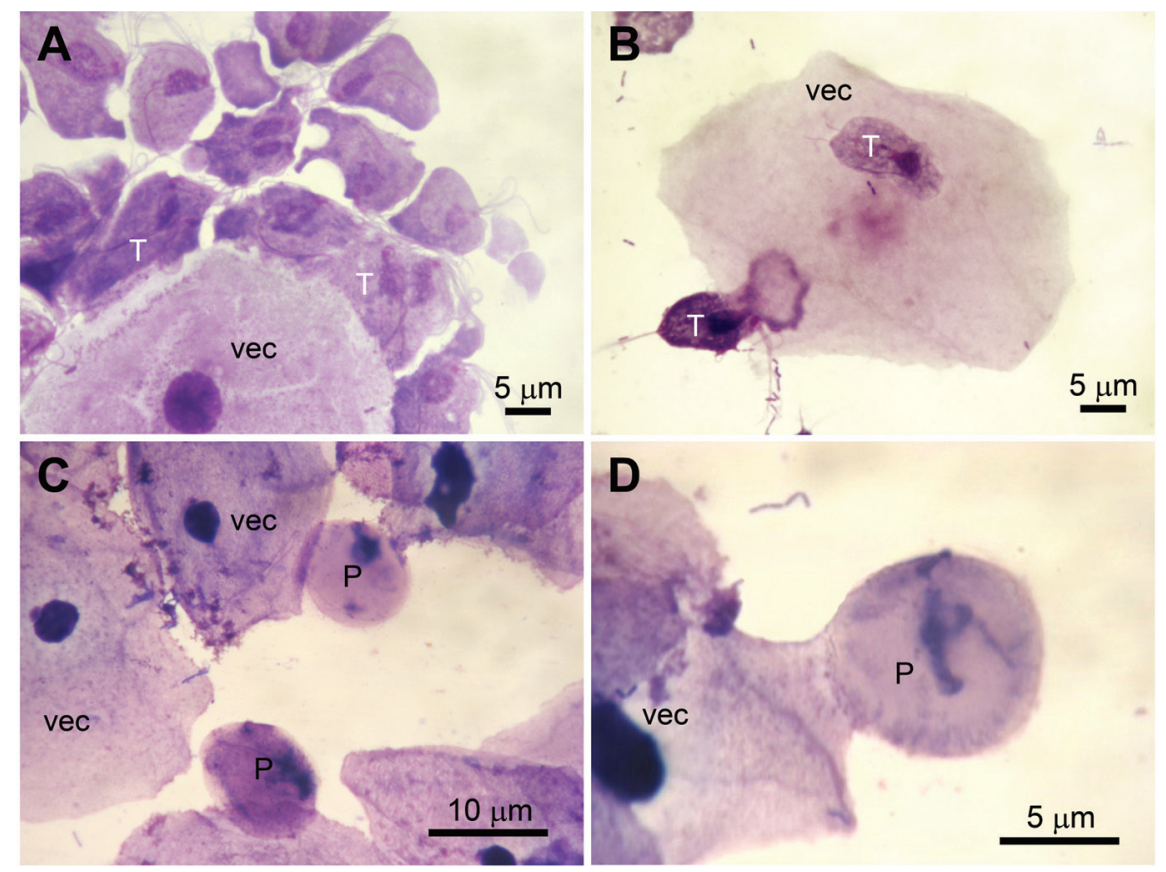

Fig. 8: interaction of Trichomonas vaginalis with VECs. Light microscopy of trophozoites-VEC interactions (A, B) or iron-depletion induced pseudocyst-VEC interactions (C, D). Samples were stained with Panotico LB kit ${ }^{\circledR}$. T: trophozoite; P: pseudocyst. 
A.

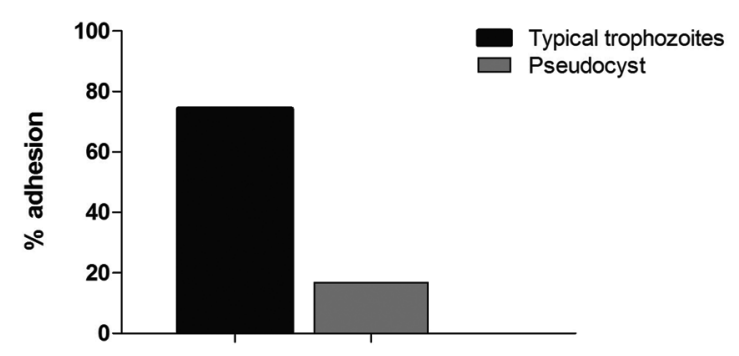

B.

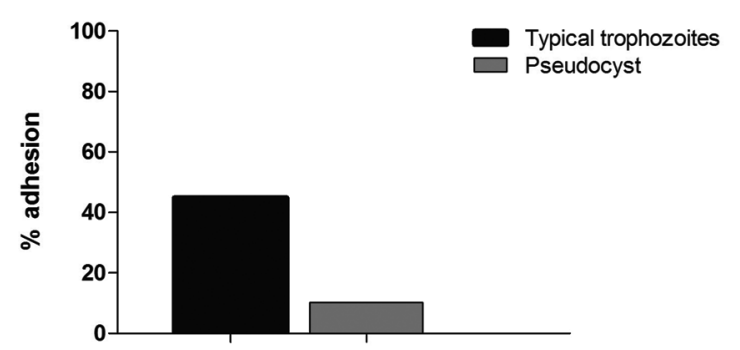

Fig. 9: percentage of adhesion of Trichomonas vaginalis trophozoites and iron-depletion induced pseudocysts to epithelial cells. The figure shows the rate of adhesion of trophozoites or iron-depletion induced pseudocysts to HeLa cells (A) or VECs (B).

laboratory analysis of clinical specimens. Searching for pseudocyst forms should also be considered during the diagnosis of trichomoniasis by wet-mount microscopic examination or by culturing methods.

\section{ACKNOWLEDGEMENTS}

To Plataforma de Microscopia Eletrônica, IOC FIOCRUZ, for technical assistance.

\section{AUTHORS' CONTRIBUTION}

JBJ designed the study; GDL, LSV, ETM, NSF, CLFC and JFP performed the experimental work; FOOJ, CB, FCSF, PC and JBJ analysed the data; GDL, PC and JBJ prepared the manuscript. All the authors read and approved the final manuscript.

\section{REFERENCES}

Abonyi A. Examination of nonflagellate and flagellate round forms of Trichomonas vaginalis by transmission electron microscopy. Appl Parasitol. 1995; 36(4): 303-10.

Afzan MY, Suresh K. Pseudocyst forms of Trichomonas vaginalis from cervical neoplasia. Parasitol Res. 2012; 111(1): 371-81.

Alderete JF. Iron modulates phenotypic variation and phosphorylation of P270 in double-stranded RNA virus-infected Trichomonas vaginalis. Infect Immun. 1999; 67(8): 4298-302.

Álvarez-Sánchez ME, Solano-González E, Yañez-Gómez C, Arroyo R. Negative iron regulation of the CP65 cysteine proteinase cytotoxicity in Trichomonas vaginalis. Microbes Infect. 2007; 9(14-15): 1597-605.

Beltrán NC, Horváthová L, Jedelský PL, Sedinová M, Rada P, Macinciková $\mathrm{M}$, et al. Iron-induced changes in the proteome of Trichomonas vaginalis hydrogenossomes. PLoS ONE. 2013; 8(5): e65148.

Benchimol M, Batista C, de Souza W. Fibronectin- and laminin Mediated endocytic activity in the parasitic protozoa Trichomonas vaginalis and Tritrichomonas foetus. J Submicr Cytol and Pathol. 1990; 22(1): 39-45.

Benchimol M, Cunha e Silva NL, Elias CA, de Souza W. Tritrichomonas foetus: ultrastructure and cytochemistry of endocytosis. Exp Parasitol. 1986; 62(3): 405-15.

Breuer W, Epsztejn S, Cabantchik ZI. Iron acquired from transferring by $\mathrm{K} 562$ cells is delivered into a cytoplasmatic pool of chelatable iron (II). J Biol Chem. 1995; 270(41): 24209-15.

Cárdenas-Guerra RE, Arroyo R, de Andrade IR, Benchimol M, Ortega-López J. The iron-induced cysteine proteinase TvCP4 plays a key role in Trichomonas vaginalis haemolysis. Microbes Infect. 2013; 15(13): 959-68.
Castro C, Menna-Barreto RF, Fernandes NS, Sabóia-Vahia L, DiasLopes G, Britto C, et al. Iron-modulated pseudocyst formation in Tritrichomonas foetus. Parasitology. 2016; 143(8): 1034-42.

da Costa RF, Benchimol M. The effect of drugs on cell structure of Tritrichomonas foetus. Parasitol Res. 2004; 92(2): 159-70.

de Jesus JB, Cuervo P, Junqueira M, Britto C, Silva-Filho FC, Soares MJ. A further proteomic study on the effect of iron in the human pathogen Trichomonas vaginalis. Proteomics. 2007; 7(12): 1961-72.

de Jesus JB, Ferreira MA, Cuervo P, Britto C, Silva-Filho FC, MeyerFernandes JR. Iron modulates ecto-phosphohydrolases activities in pathogenic trichomonads. Parasitol Int. 2006; 55(4): 285-90.

de Jesus JB, Vannier-Santos MA, Britto C, Godefroy P, Silva-Filho FC, Pinheiro AA, et al. Trichomonas vaginalis virulence against epithelial cells and morphological variability: the comparison between a well-established strain and a fresh isolate. Parasitol Res. 2004; 93(5): 369-77.

Granger BL, Warwood SJ, Benchimol M, de Souza W. Transient invagination of flagella by Tritrichomonas foetus. Parasitol Res. 2000; 86(9): 699-709.

Hernández-Cuevas NA, Weber C, Hon CC, Guillen N. Gene expression profiling in Entamoeba histolytica identifies key components in iron uptake and metabolism. PLoS ONE. 2014; 9(9): e107102.

Hsu HM, Lee Y, Indra D, Wei SY, Liu HW, Chang LC, et al. Ironinducible nuclear translocation of a Myb3 transcription factor in the protozoan parasite Trichomonas vaginalis. Eukaryot Cell. 2012; 11(12): 1441-50.

Kissinger P. Trichomonas vaginalis: a review of epidemiologic, clinical and treatment issues. BMC Infect Dis. 2015; 15: 307.

Kummer S, Hayes GR, Gilbert RO, Beach DH, Lucas JJ, Singh BN. Induction of human host cell apoptosis by Trichomonas vaginalis cysteine proteases is modulated by parasite exposure to iron. Microb Pathog. 2008; 44(3): 197-203.

Lehker MW, Alderete JF. Iron regulates growth of Trichomonas vaginalis and the expression of immunogenic trichomonad proteins. Mol Microbiol. 1992; 6(1): 123-32.

Lipman NS, Lampen N, Nguyen HT. Identification of pseudocysts of Tritrichomonas muris in Armenian hamsters and their transmission to mice. J Am Assoc Lab Anim Sci. 1999; 49(3): 313-15.

Meites E, Gaydos CA, Hobbs MM, Kissinger P, Nyirjesy P, Schwebke $\mathrm{JR}$, et al. A review of evidence-based care of symptomatic Trichomoniasis and asymptomatic Trichomonas vaginalis infections. Clin Infect Dis. 2015; 61(8): 837-48. 
Melo-Braga MB, Rocha-Azevedo B, Silva-Filho FC. Tritrichomonas foetus: the role played by iron during parasite interaction with epithelial cells. Exp Parasitol. 2003; 105(2): 111-20.

Mesquita-Rodrigues C, Menna-Barreto RFS, Sabóia-Vahia L, da Silva SAG, de Souza EM, Waghabi MC, et al. Cellular growth and mitochondrial ultrastructure of Leishmania (Viannia) braziliensis promastigotes are affected by the iron chelator 2,2-dipyridyl. PLoS Negl Trop Dis. 2013; 7(10): e2481.

Meza-Cervantez P, González-Robles A, Cardenas-Guerra RE, Ortega-López J, Saavedra E, Pineda E, et al. Pyruvate:ferredoxin oxidoreductase (PFO) is a surface-associated cell-binding protein in Trichomonas vaginalis and is involved in trichomonal adherence to host cells. Microbiology. 2011; 157(12): 3469-82.

Olmo F, Urbanoy K, Rosales MJ, Martin-Escolano R, SánchezMoreno MS, Marin C. An in vitro iron superoxide dismutase inhibitor decreases the parasitemia levels of Trypanosoma cruzi in $\mathrm{BALB} / \mathrm{c}$ mouse model during acute phase. Int J Parasitol Drugs Drug Resist. 2014; 5(3): 110-16.

Pereira-Neves A, Benchimol M. Tritrichomonas foetus: budding from multinucleated pseudocysts. Protist. 2009; 160(4): 536-51.
Pereira-Neves A, Campero CM, Martínez A, Benchimol M. Identification of Tritrichomonas foetus pseudocysts in fresh preputial secretion samples from bulls. Vet Parasitol. 2011; 175(1-2): 1-8.

Pereira-Neves A, Nascimento LF, Benchimol M. Cytotoxic effects exerted by Tritrichomonas foetus pseudocysts. Protist. 2012; 163(4): 529-43.

Pereira-Neves A, Ribeiro KC, Benchimol M. Pseudocysts in trichomonads - new insights. Protist. 2003; 154(3-4): 313-29.

Ribeiro LC, Santos C, Benchimol M. Is Trichomonas tenax a parasite or a commensal? Protist. 2015; 166(2): 196-210.

Thipubon P, Uthaipibull C, Kamchonwongpaisan S, Tipsuwan W, Richairatanakool S. Inhibitory effect of novel iron chelator, $\mathrm{N}$ acetyl-6-aminohexyl)-3-hydroxy-2-ethylpyridin-4-one (CM1) and green tea extract on growth of Plasmodium falciparum. Malar J. 2015; 14: 382.

Vanáčová S, Rasoloson D, Rázga J, Hrdý I, Kulda J, Tachezy J. Ironinduced changes in pyruvate metabolism of Tritrichomonas foetus and involvement of iron in expression of hydrogenosomal proteins. Microbiology. 2001; 147(1): 53-62.

WHO - World Health Organization. Global incidence and prevalence of selected sexually transmitted infections - 2008. Geneva: WHO; 2012. 20 pp. 